\title{
Adaptive Multivariable Super-Twisting Sliding Mode Controller and Disturbance Observer Design for Hypersonic Vehicle
}

\author{
Wenru Fan ${ }^{1}$ and Bailing Tian ${ }^{2}$ \\ ${ }^{1}$ Aeronautical Automation College, Civil Aviation University of China, Tianjin 300300, China \\ ${ }^{2}$ School of Electric and Automation Engineering, Tianjin University, Tianjin 300072, China \\ Correspondence should be addressed to Bailing Tian; bailing_tian@tju.edu.cn
}

Received 10 May 2016; Accepted 14 July 2016

Academic Editor: Mohamed Djemai

Copyright (c) 2016 W. Fan and B. Tian. This is an open access article distributed under the Creative Commons Attribution License, which permits unrestricted use, distribution, and reproduction in any medium, provided the original work is properly cited.

\begin{abstract}
A multivariable super-twisting sliding mode controller and disturbance observer with gain adaptation, chattering reduction, and finite time convergence are proposed for a generic hypersonic vehicle where the boundary of aerodynamic uncertainties exists but is unknown. Firstly, an input-output linearization model is constructed for the purpose of controller design. Then, the sliding manifold is designed based on the homogeneity theory. Furthermore, an integrated adaptive multivariable super-twisting sliding mode controller and disturbance observer are designed in order to achieve the tracking for step changes in velocity and altitude. Finally, some simulation results are provided to verify the effectiveness of the proposed method.
\end{abstract}

\section{Introduction}

Hypersonic vehicles are intended to be a reliable and costeffective technology for access to space. During the past decades, a considerable effort has been made by the US Air Force and NASA to further their development. However, the design of control system for hypersonic vehicles is a challenging work due to high speed flight which causes the vehicle to be very sensitive of changes in flight conditions. In addition, the vehicle suffers from severe aerodynamic uncertainties which make the controller design more difficult. Despite the difficulties mentioned above, much effort has been done to develop advanced control technologies for hypersonic vehicle in the past few years.

For the design of control systems for hypersonic vehicles based on linearized dynamical models, several results are available in the literature. For example, Schmidt employed classic and multivariable linear control approach [1] and Groves et al. applied Linear Quadratic Regulator (LQR) technique [2], while Hughes used $H_{\infty}$ as well as Linear Parameter Varying (LPV) method [3], and Sigthorsson et al. used implicit model-following control methods [4] to design controller for a linearized hypersonic vehicle model at a specified trim condition. Based on the methods, flight control design is carried out by linearizing the system at a series of operating points and designing separate controllers at each of these points. Finally, the overall flight control system is realized in the philosophy of gain scheduling where the individual gains are interpolated online with respect to some meaningful parameters such as dynamic pressure and Mach number. However, the number of required gains to be designed and scheduled within the controller becomes very large in order to cover different flight missions. In addition, the method involves the lack of guaranteed global robustness, performance, and especially stability [5].

As far as nonlinear control design is concerned, the back-stepping [6], trajectory linearization control [7], robust inversion-based technique [8], sequential loop closure [9], and adaptive control [10] have been developed for hypersonic vehicle in order to improve the robustness and control precision. Although many nonlinear control techniques have been proposed during the last decades, the sliding mode control (SMC) remains a key choice in handing the system with bounded uncertainties and disturbances due to its robustness [11-13]. However, one of the issues of traditional SMC is control chattering caused by high-frequency control switching which restricts its application in real system. $\mathrm{Xu}$ et al. [14] combined the adaptive technique with the SMC to design an adaptive sliding mode controller for a generic hypersonic vehicle to track the step commands in 
velocity and altitude while requiring limited state information. In the method, the behavior of continuous control is achieved via boundary layer technique at the sacrifice of robustness and tracking accuracy to external disturbances and model uncertainties. In addition, it should be noted that the control law designed based on this method is asymptotically stable which means that the convergence rate is at best exponential with infinite settling time. It is obvious that the control law with finite time convergence is more desirable due to the fact that the closed-loop system under finite time control usually demonstrates higher accuracy and better disturbance rejection properties [15]. Preserving the SMC features, the high order sliding mode (HOSM) technique is capable of removing the chattering and improving accuracy and convergent rate [16]. A potential disadvantage of the HOSM to be used in hypersonic vehicle is that the upper bound of uncertainty has to be known exactly in advance. However, it may be difficult to obtain the boundary prior to fly for hypersonic vehicle due to its complex characteristics. Subsequently, the adaptive high order sliding mode control schemes $[17,18]$ are proposed for hypersonic vehicle where an adaptive law is designed to estimate the uncertainty's upper bound. In the method, it only requires that the uncertainty is bounded which is a mild assumption for most practical system. However, these methods mentioned above are worst-case-based design and involve the issue of overconservation. Disturbance observerbased control methods provide an effective way to address the issue [19-21]. In the methods, a nonlinear disturbance observer (NDOB) is employed to estimate the uncertainty as well as external disturbance and then a nominal controller is designed for hypersonic vehicle. As a result, the methods obtain not only promising robustness and disturbance rejection performance but also nominal performance recovery. Nevertheless, an assumption of the upper bound of uncertainty has to be known in advance in order to successfully design the NDOB. As noted earlier, this condition may not be available in practice. The first motivation of the research is to propose a practical robust control scheme which is independent on the upper boundary of uncertainty. In addition, it should preserve the advantages of disturbance observerbased methods in $[19,20]$, such as disturbance rejection, nominal performance recovery, and finite time convergence. Finally, it should be noted that all the methods mentioned above are designed based on single-variable control scheme where only one control input is included. To design multivariable flight control system, the single-variable control scheme is used via decoupling the system into multiple singlevariable systems. As pointed in [22], if the multivariable control system is designed directly using the multivariable control scheme, it would have improved chattering reduction property which is the second motivation of the research.

The paper is organized as follows. In Section 2 the vehicle model is introduced and the control objective is stated. The design and stability analysis of adaptive multivariable supertwisting sliding mode controller and disturbance are presented in Section 3. Finally, simulation results are discussed in Section 4, and conclusions are provided in Section 5.

\section{Problem Formulation}

2.1. Hypersonic Vehicle Model. The longitudinal dynamic model of a generic hypersonic vehicle developed at NASA Langley Research Center is used here depicted by [14]

$$
\begin{aligned}
& \dot{v}=\frac{T \cos \alpha-D}{m}-\frac{\mu \sin \gamma}{r^{2}}, \\
& \dot{\gamma}=\frac{L+T \sin \alpha}{m V}-\frac{\left(\mu-v^{2} r\right) \cos \gamma}{v r^{2}} \\
& \dot{h}=v \sin \gamma \\
& \dot{\alpha}=q-\dot{\gamma}, \\
& \dot{q}=\frac{M_{y y}}{I_{y y}} \\
& \ddot{\phi}=-2 \varsigma \omega_{n} \dot{\phi}-\omega_{n}^{2} \phi+\omega_{n}^{2} \phi_{c},
\end{aligned}
$$

where $\phi_{c}$ is control input, fuel equivalence ratio. The state vector for system (1) and (2) is $\mathbf{x}=[v, \gamma, h, \alpha, q, \phi, \dot{\phi}]^{T}$ which denotes the velocity, flight path angle (FPA), altitude, angle of attack (AOA), pitch rate, and throttle setting and its time derivative, respectively. Equation (2) is second-order engine dynamics with damping coefficient $\varsigma=0.7$ and natural frequency $\omega_{n}=5$. The variable $r=h+\operatorname{Re}$ denotes the distance between the vehicle and the center of earth. At the trimmed conditions, $v=15060 \mathrm{ft} / \mathrm{s}, h=110000 \mathrm{ft}, \alpha=0.0315 \mathrm{rad}$, $q=0 \mathrm{rad}$, and $\gamma=0 \mathrm{rad}$, the lift force $L$, drag force $D$, thrust force $T$, and pitch moment $M_{y y}$ are calculated as

$$
\begin{aligned}
L & =\frac{1}{2} \rho v^{2} S C_{L}(\alpha), \\
D & =\frac{1}{2} \rho v^{2} S C_{D}(\alpha), \\
T & =\frac{1}{2} \rho v^{2} S C_{T}(\phi), \\
M_{y y} & =\frac{1}{2} \rho v^{2} S c\left[C_{M}(\alpha)+C_{M}\left(\delta_{e}\right)+C_{M}(q)\right]
\end{aligned}
$$

with

$$
\begin{aligned}
C_{L}(\alpha) & =0.6203 \alpha, \\
C_{D}(\alpha) & =0.6450 \alpha^{2}+0.0043378 \alpha+0.003772 \alpha, \\
C_{M}(\alpha) & =-0.035 \alpha^{2}+0.036617 \alpha+5.3261 \times 10^{-6}, \\
C_{M}\left(\delta_{e}\right) & =0.0292\left(\delta_{e}-\alpha\right), \\
C_{M}(q) & =\frac{c}{2 v} q\left(-6.796 \alpha^{2}+0.3015 \alpha-0.2289\right), \\
C_{T}(\phi) & = \begin{cases}0.02576 \phi & \text { if } \phi \leq 1 \\
0.0224+0.00336 \phi & \text { if } \phi>1\end{cases}
\end{aligned}
$$

here $\delta_{e}$ is elevator deflection. 
2.2. Control Objective. The objective of the research is to determine the control inputs $\mathbf{u}=\left[\phi_{c}, \delta_{e}\right]^{T}$ which makes the system output track the desired commands, velocity $v_{d}$, and altitude $h_{d}$, in finite time in the presence of the following bounded but unknown perturbations:

$$
\begin{aligned}
& \left|\Delta_{C_{L}}\right| \leq 0.2 C_{L}, \\
& \left|\Delta_{C_{D}}\right| \leq 0.2 C_{D}, \\
& \left|\Delta_{C_{T}}\right| \leq 0.2 C_{T}, \\
& \left|\Delta_{C_{M}}\right| \leq 0.2\left[C_{M}(\alpha)+C_{M}\left(\delta_{e}\right)+C_{M}(q)\right] .
\end{aligned}
$$

\section{Adaptive Multivariable Super-Twisting Sliding Mode Control Scheme}

3.1. Preliminary. In the subsection, several useful lemmas to be used in the design of controller and disturbance observer for hypersonic vehicle are recalled.

Lemma 1 (see [23]). Consider a non-Lipschitz system in the form of $\dot{\mathbf{x}}=f(\mathbf{x}), f(0)=0$, and suppose that there exist a Lyapunov function $V(\mathbf{x})$ and real numbers $\lambda_{1}>0, \lambda_{2}>0$, and $a \in(0,1)$, such that $V(\mathbf{x})$ is positive for any nonzero $\mathbf{x}$ and inequality $\dot{V}(\mathbf{x})+\lambda_{1} V(\mathbf{x})^{a}+\lambda_{2} V(\mathbf{x}) \leq 0$ holds. Then, the origin is fast finite time stable and the settling time, depending on the initial state $\mathbf{x}(0)=\mathbf{x}_{0}$, given by $T\left(\mathbf{x}_{0}\right) \leq\left(1 / \lambda_{1}(1-\right.$ a)) $\ln \left(\left(\lambda_{1} V^{1-a}\left(\mathbf{x}_{0}\right)+\lambda_{2}\right) / \lambda_{2}\right)$.

Lemma 2 (see [24]). Suppose that there exist constant $v \in$ $(0,1)$ and positive constants $k_{1}, \ldots, k_{n}$ such that polynomial $s^{n}+k_{n} s^{n-1}+\cdots+k_{2} s^{n}+k_{1}$ is Hurwitz. Then, the integrator chain system $\dot{x}_{1}=x_{2}, \dot{x}_{2}=x_{3}, \ldots, \dot{x}_{n}=u$ is finite time stable under the feedback $u=-k_{1}\left|x_{1}\right|^{v_{1}} \operatorname{sign}\left(x_{1}\right)-\cdots-k_{n}\left|x_{n}\right|^{v_{n}} \operatorname{sign}\left(x_{n}\right)$, where sign denotes the signum function and $v_{i}(i=2,3, \ldots, n)$ satisfies $v_{i-1}=v_{i} v_{i+1} /\left(2 v_{i+1}-v_{i}\right)$ with $v_{n}=v, v_{n+1}=1$.

3.2. Main Results. Inspired from the research in [22], the single-variable control scheme $[25,26]$ is extended to the following multivariable super-twisting sliding mode control scheme.

Theorem 3. Consider the following multivariable system:

$$
\begin{aligned}
& \dot{\mathbf{z}}_{1}=-K_{1}(t) \frac{\mathbf{z}_{1}}{\left\|\mathbf{z}_{1}\right\|^{1 / 2}}-K_{2}(t) \mathbf{z}_{1}+\mathbf{z}_{2}, \\
& \dot{\mathbf{z}}_{2}=-K_{3}(t) \frac{\mathbf{z}_{1}}{\left\|\mathbf{z}_{1}\right\|}-K_{4}(t) \mathbf{z}_{1}-\Delta,
\end{aligned}
$$

where $\mathbf{z}_{1}, \mathbf{z}_{2} \in R^{m}$ and $K_{i}(t)(i=1,2,3,4)$ is adaptive gain to be developed soon and suppose that the perturbation $\Delta$ satisfies the condition $\|\Delta\| \leq \delta$, where the finite boundary $\delta>0$ exists but is unknown. Then, one has the following claims.
(1) If the gain $K_{i}(t)$ in (6) is designed as

$$
\begin{aligned}
K_{1}(t) & =k_{1} \sqrt{L(t)}, \\
K_{2}(t) & =k_{2} L(t), \\
K_{3}(t) & =k_{3} L(t), \\
K_{4}(t) & =k_{4} L^{2}(t), \\
\dot{L}(t) & = \begin{cases}k & \text { if }\left\|\mathbf{z}_{1}\right\| \neq 0 \\
0 & \text { otherwise }\end{cases}
\end{aligned}
$$

with positive scalar $k_{i}(i=1,2,3,4)$ satisfying

$$
9 k_{1}^{2} k_{2}^{2}+8 k_{2}^{2} k_{3}<4 k_{3} k_{4},
$$

then vectors $\mathbf{z}_{1}$ and $\mathbf{z}_{2}$ converge to zero in finite time.

(2) For any positive constants $K_{i}(t)>0$, the states of system (6) are globally bounded for any bounded perturbation $\Delta$.

(3) The vectors $\mathbf{z}_{1}$ and $\mathbf{z}_{2}$ converge to zero for any positive parameter $K_{i}(t)>0$ if there is no perturbation for system (6), which means $\Delta=\mathbf{0}$ in (6).

Proof. The first claim is a direct result of our recent work [27]. Following the conclusion in [27], it follows that there exists a continuous and positive definite Lyapunov function

$$
\begin{aligned}
V= & 2 k_{3}\left\|\xi_{1}\right\|^{2}+k_{4}\left\|\xi_{2}\right\|^{2}+0.5\left\|\xi_{3}\right\|^{2} \\
& +0.5\left\|k_{1} \xi_{1}+k_{2} \xi_{2}-\xi_{3}\right\|^{2}
\end{aligned}
$$

for system (6) such that

$$
\dot{V} \leq-\underbrace{\left(L(t) \pi_{1}-\delta \pi_{4}\right)}_{\gamma_{1}} V^{1 / 2}-\underbrace{\left(L(t) \pi_{2}-\frac{\dot{L}(t)}{L(t)} \pi_{3}\right)}_{\gamma_{2}} V
$$

with $\boldsymbol{\xi}_{1}=\left[L /\left\|\mathbf{z}_{1}\right\|\right]^{1 / 2} \mathbf{z}_{1}, \boldsymbol{\xi}_{2}=L \mathbf{z}_{1}, \boldsymbol{\xi}_{3}=\mathbf{z}_{3}$, and some bounded constants $\pi_{1}, \pi_{2}, \pi_{3}$, and $\pi_{4}$ provided in [27]. From the definition of adaptive law in (7), it can be observed that the items $\gamma_{1}$ and $\gamma_{2}$ in (10) will be positive in finite time for arbitrary constants $\pi_{1}, \pi_{2}, \pi_{3}$, and $\pi_{4}$ and boundary perturbation $\delta$ since $\dot{L}(t) \geq 0$. After that, one has $\dot{V}+\gamma_{1} V^{1 / 2}+$ $\gamma_{2} V \leq 0$ implying $\boldsymbol{\varsigma}=\left[\boldsymbol{\varsigma}_{1}, \boldsymbol{\varsigma}_{2}, \boldsymbol{\varsigma}_{3}\right]^{T} \rightarrow 0$ equivalently $\mathbf{z}_{1}, \mathbf{z}_{2} \rightarrow$ 0 in finite time which proves the first claim of Theorem 3 .

In order to prove the second claim, we can set $L(t)=1$, which implies $\dot{L}(t) \equiv 0$. In this case, (10) is reduced to

$$
\dot{V} \leq-\pi_{1} V^{1 / 2}+\delta \pi_{4} V^{1 / 2}-\pi_{2} V .
$$

Next, the item $-\pi_{2} V$ in (11) can be used to dominate $\delta \pi_{4} V^{1 / 2}$ for sufficient large $V$. To this end, we rewrite inequality (11) as

$$
\dot{V} \leq-\pi_{1} V^{1 / 2}-\left(\theta \pi_{2} V^{1 / 2}-\delta \pi_{4}\right) V^{1 / 2}-(1-\theta) \pi_{2} V,
$$

where $\theta$ is a positive scalar satisfying $0<\theta<1$. It is obvious that

$$
\dot{V} \leq-\pi_{1} V^{1 / 2}-(1-\theta) \pi_{2} V, \quad \forall V \geq \underbrace{\left[\frac{\delta \pi_{4}}{\theta \pi_{2}}\right]^{2}}_{\Upsilon} .
$$


In view of inequality (13) and theorem 4.18 in [28], it can be concluded that the solutions starting in the set $\Xi_{1}=$ $\{V \leq \Upsilon\}$ with $\Upsilon$ in (13) will remain therein for all future time since $\dot{V}$ is negative on the boundary $\|V\|=\Upsilon$. On the other hand, if the solutions start outside the set $\Xi_{1}, V$ will decrease monotonically until the solution enters the set $\Xi_{1}$. After that, the solution cannot leave the set $\Xi_{1}$. Hence, the states of system (6) are globally bounded for any bounded perturbation $\Delta$.

With respect to the third claim, we can set $\delta \equiv 0, L(t) \equiv 1$, and $\dot{L}(t)=0$. Then (11) can be reduced to $\dot{V} \leq-\pi_{1} V^{1 / 2}-\pi_{2} V$. It follows from Lemma 2 that the vectors $\mathbf{z}_{1}$ and $\mathbf{z}_{2}$ converge to zero for any positive parameter $K_{i}(t)>0$ if $\boldsymbol{\Delta}=\mathbf{0}$ holds in (6).

Corollary 4. Consider the following general multivariable uncertainty system:

$$
\dot{\mathbf{x}}=f(\mathbf{x})+\mathbf{u}+\Delta_{f}(\mathbf{x})
$$

where $\mathbf{x} \in R^{m}, \mathbf{u} \in R^{m}$ are state and control vector and $f(\mathbf{x}) \in R^{m}, \Delta_{f}(\mathbf{x}) \in R^{m}$ represent nominal and uncertainty parts, respectively. It is assumed that uncertainty $\Delta_{f}(\mathbf{x})$ is continuously differentiable with $\left\|\dot{\Delta}_{f}(\mathbf{x})\right\| \leq \delta$, where $\delta$ exists but is unknown. Furthermore, the following conclusions can be obtained.

(1) If an adaptive multivariable disturbance observer is designed,

$$
\begin{aligned}
& \dot{\mathbf{z}}_{1}=-K_{1}(t) \frac{\mathbf{e}_{1}}{\left\|\mathbf{e}_{1}\right\|^{1 / 2}}-K_{2}(t) \mathbf{e}_{1}+f(\mathbf{x})+\mathbf{u}+\mathbf{z}_{2}, \\
& \dot{\mathbf{z}}_{2}=-K_{3}(t) \frac{\mathbf{e}_{1}}{\left\|\mathbf{e}_{1}\right\|}-K_{4}(t) \mathbf{e}_{1}
\end{aligned}
$$

with $\mathbf{e}_{1}=\mathbf{z}_{1}-\mathbf{x}$ and $K_{i}(t)$ being chosen according to (7) and (8). Then, $\boldsymbol{\Delta}_{f}(\mathbf{x})$ can be estimated through $\mathbf{z}_{2}$ in finite time.

(2) If multivariable controller is designed,

$$
\begin{aligned}
\mathbf{u} & =-f(\mathbf{x})-\widehat{\Delta}_{f}(\mathbf{x})-k_{1} \frac{\mathbf{x}}{\|\mathbf{x}\|^{1 / 2}}-k_{2} \mathbf{x}+\boldsymbol{\omega}_{2}, \\
\dot{\boldsymbol{\omega}}_{2} & =-k_{3} \frac{\mathbf{x}}{\|\mathbf{x}\|}-k_{4} \mathbf{x},
\end{aligned}
$$

where $\widehat{\boldsymbol{\Delta}}_{f}(\mathbf{x})=\mathbf{z}_{2}$ is disturbance estimation generated from (15) and $k_{i}>0(i=1,2,3,4)$ is arbitrary positive constant. Then, $\mathbf{x}$ in (14) converges to zero in finite time under the controller (16) and disturbance observer (15).

Proof. Combining (14) and the definitions $\mathbf{e}_{1}=\mathbf{z}_{1}-\mathbf{x}$ and $\mathbf{e}_{2}=\mathbf{z}_{2}-\Delta_{f}(\mathbf{x}),(15)$ is converted into

$$
\begin{aligned}
& \dot{\mathbf{e}}_{1}=-K_{1}(t) \frac{\mathbf{e}_{1}}{\left\|\mathbf{e}_{1}\right\|^{1 / 2}}-K_{2}(t) \mathbf{e}_{1}+\mathbf{e}_{2}, \\
& \dot{\mathbf{e}}_{2}=-K_{3}(t) \frac{\mathbf{e}_{1}}{\left\|\mathbf{e}_{1}\right\|}-K_{4}(t) \mathbf{e}_{1}-\dot{\Delta}_{f}(\mathbf{x}) .
\end{aligned}
$$

Based on Theorem 3, it can be concluded that the estimation error vectors $\mathbf{e}_{1}$ and $\mathbf{e}_{2}$ converge to zero in finite time if the parameters $K_{i}(t)$ are chosen according to (7) and (8), which implies that $\Delta_{f}(\mathbf{x})$ can be reconstructed via $\mathbf{z}_{2}$ in finite time. This completes the proof of the first part of Corollary 4. Furthermore, the closed-loop system (14) under controller (16) and disturbance observer (15) has the following form:

$$
\begin{aligned}
\dot{\mathbf{x}} & =-k_{1} \frac{\mathbf{x}}{\|\mathbf{x}\|^{1 / 2}}-k_{2} \mathbf{x}+\boldsymbol{\omega}_{2}+\mathbf{e}_{2}, \\
\dot{\boldsymbol{\omega}}_{2} & =-k_{3} \frac{\mathbf{x}}{\|\mathbf{x}\|}-k_{4} \mathbf{x} .
\end{aligned}
$$

For convenience, a new vector $\overline{\boldsymbol{\omega}}_{2}=\boldsymbol{\omega}_{2}+\mathbf{e}_{2}$ is introduced so that (18) can be rewritten as $\dot{\mathbf{x}}=-k_{1}\left(\mathbf{x} /\|\mathbf{x}\|^{1 / 2}\right)-k_{2} \mathbf{x}+\overline{\boldsymbol{\omega}}_{2}, \dot{\overline{\boldsymbol{\omega}}}_{2}=$ $-k_{3}(\mathbf{x} /\|\mathbf{x}\|)-k_{4} \mathbf{x}+\dot{\mathbf{e}}_{2}$. It follows from the second conclusion in Theorem 3 that this system is input-to-state stable with $\dot{\mathbf{e}}_{2}$ viewed as input. From estimation error dynamics in (17), it can be observed that $\dot{\mathbf{e}}_{2}$ is bounded. Therefore, the state $\mathbf{x}$ in (18) is bounded for arbitrary bounded $\dot{\mathbf{e}}_{2}$. Furthermore, it follows from the first conclusion in Theorem 3 that there exists a finite time $t_{1}$ such that the estimation error $\mathbf{e}_{2}(t)=0$ for $t \geq t_{1}$. After that, system (18) is reduced to nominal system: $\dot{\mathbf{x}}=-k_{1}\left(\mathbf{x} /\|\mathbf{x}\|^{1 / 2}\right)-k_{2} \mathbf{x}+\omega_{2}, \omega_{2}=-k_{3}(\mathbf{x} /\|\mathbf{x}\|)-$ $k_{4} \mathbf{x}$ with bounded initial value $\mathbf{x}\left(t_{1}\right)$. Then, based on the third conclusion in Theorem 3 , it can be seen that vector $\mathbf{x}$ converges to zero in finite time for arbitrary positive scalar $k_{i}>0(i=1,2,3,4)$. This completes the proof of the second part of Corollary 4.

Remark 5. In practice $\left\|\mathbf{z}_{1}\right\|$ cannot be zero exactly. Therefore, $L(t)$ will increase unboundedly. To avoid this drawback, the adaptive law in (7) can be modified as $\dot{L}(t)=k$ if $\left\|\mathbf{z}_{1}\right\| \geq$ $\varepsilon$; otherwise $\dot{L}(t)=0$, where $\varepsilon$ is a positive scalar. The idea of the adaptive law can be interpreted as follows: the gain increases when $\left\|\mathbf{z}_{1}\right\|$ unacceptably deviates from zero and it stops increasing when $\left\|\mathbf{z}_{1}\right\|$ is driven into the regime $\left\|\mathbf{z}_{1}\right\|<\varepsilon$.

Remark 6. The introduction of an adaptive disturbance observer in control scheme has two reasons. First, disturbance observer is able to provide estimation for uncertainties and then the estimated value can be used in control input to compensate the actual uncertainties in plant. In this case, the baseline controller with small control gains can be used to stabilize the closed-loop system, which implies the properties of chattering reduction and nominal performance recovery are obtained (see [21]). Furthermore, the gain adaptation is used in disturbance observer in order to avoid dependency on upper boundary of uncertainty which may be difficult to obtain in practice.

3.3. Controller and Disturbance Observer Design. It follows from the results in [14] that the input-output linearized model 
can be obtained via differentiating velocity and altitude three and four times, respectively, depicted by

$$
\left[\begin{array}{c}
\ddot{v} \\
h^{(4)}
\end{array}\right]=\left[\begin{array}{l}
F_{1 v} \\
F_{1 h}
\end{array}\right]+\left[\begin{array}{ll}
G_{11} & G_{12} \\
G_{22} & G_{23}
\end{array}\right]\left[\begin{array}{l}
\phi \\
\delta_{e}
\end{array}\right]+\left[\begin{array}{l}
\Delta_{F_{v}} \\
\Delta_{F_{h}}
\end{array}\right],
$$

where $\Delta_{F_{v}}$ and $\Delta_{F_{h}}$ are bounded perturbation induced by model parameters uncertainties. All the parameters in (19) can be referred to [14]. For brevity, the details are omitted. Furthermore, the sliding mode manifolds for velocity and altitude are designed as

$$
\begin{aligned}
& s_{v}=\ddot{e}_{v}+\int \underbrace{\left[k_{1 v}\left|e_{v}\right|^{v_{1 v}} \operatorname{sign}\left(e_{v}\right)+k_{2 v}\left|\dot{e}_{v}\right|^{v_{2 v}} \operatorname{sign}\left(\dot{e}_{v}\right)+k_{3 v}\left|\ddot{e}_{v}\right|^{v_{3 v}} \operatorname{sign}\left(\ddot{e}_{v}\right)\right]}_{F_{1 v}}, \\
& s_{h}=\ddot{e}_{h}-\int \underbrace{\left[k_{1 h}\left|e_{h}\right|^{v_{1 h}} \operatorname{sign}\left(e_{h}\right)+k_{2 h}\left|\dot{e}_{h}\right|^{v_{2 h}} \operatorname{sign}\left(\dot{e}_{h}\right)+k_{3 h}\left|\ddot{e}_{h}\right|^{v_{3 h}} \operatorname{sign}\left(\ddot{e}_{h}\right)+k_{4 h}\left|\ddot{e}_{h}\right|^{v_{4 h}} \operatorname{sign}\left(\ddot{e}_{h}\right)\right]}_{F_{1 h}},
\end{aligned}
$$

where $e_{v}=v-v_{d}$ and $e_{h}=h-h_{d}$. It follows the results in Lemma 2 that $e_{v}$ and $e_{h} \rightarrow 0$ in finite time if the conditions $s_{v}=s_{h}=0$ hold and the sliding manifold parameters $v_{i v}(i=$ $1,2,3)$ and $v_{j h}(j=1,2,3,4)$ are chosen properly. To this end, taking the derivative of $s_{v}$ and $s_{h}$ along the trajectories in (20) yields

$$
\begin{aligned}
\underbrace{\left[\begin{array}{c}
\dot{\boldsymbol{s}}_{v} \\
\dot{s}_{h}
\end{array}\right]}_{\mathbf{s}}=\underbrace{\left[\begin{array}{c}
F_{1 v}+F_{2 v}-\ddot{v}_{d} \\
F_{1 h}+F_{2 h}-h_{d}^{(4)}
\end{array}\right]}_{\mathbf{F}}+\underbrace{\left[\begin{array}{cc}
G_{11} & G_{12} \\
G_{21} & G_{22}
\end{array}\right]\left[\begin{array}{l}
\varphi \\
\delta_{e}
\end{array}\right]}_{\mathbf{U}} \\
+\underbrace{\left[\begin{array}{c}
\Delta_{F_{v}} \\
\Delta_{F_{h}}
\end{array}\right] .}_{\Delta}
\end{aligned}
$$

The uncertainties vector $\Delta$ can be interpreted as total perturbations which may be caused by model parameter uncertainty and unknown external disturbance. In the simulation, $\Delta$ is induced by uncertainties in (5). Furthermore, it is assumed that $\Delta$ is continuously differentiable such that $\|\dot{\Delta}\| \leq$ $\delta$ holds, where $\delta$ is a finite value but unknown. In fact, $\Delta$ is a function of system states. In real applications, the derivatives of system states should be bounded in order to guarantee safe and stable flight for vehicle, which can be confirmed through the results to be provided in Figures 2(d) and 2(e). Consequently, it is a mild assumption. It is obvious that system (21) can be considered as special case for the general multivariable system in (14). Consequently, the adaptive multivariable controller and disturbance observer can be easily designed based on Corollary 4 . For brevity, the details are omitted here.

Remark 7. It should be noted that the missing derivatives of $e_{v}$ and $e_{h}$ in sliding manifolds and control law can be estimated online using an arbitrary order robust finite time convergent differentiator [29].

\section{Simulation Results Analysis}

4.1. Parameters Setting. The parameters used in the simulation are summarized as follows. The sliding manifold parameters in (20) are taken as $k_{1 v}=k_{1 h}=0.5, k_{2 v}=k_{2 h}=$ $1.4, k_{3 v}=2.5, k_{3 h}=1.8, k_{4 h}=2.5$, and $v_{3 v}=v_{4 h}=0.7$.
The other parameters $v_{i v}(i=1,2)$ and $v_{j h}(j=1,2,3)$ are calculated according to Lemma 2 . The parameters used in disturbance observer and controller are chosen as $k_{1}=k_{3}=$ $0.2, k_{2}=k_{4}=0.05$, and $k=0.01$. The tolerance constant $\varepsilon$ mentioned in Remark 5 is 0.001 . The step commands for velocity $100 \mathrm{ft} / \mathrm{s}$ and altitude $1000 \mathrm{ft}$ are used as the reference commands to be tracked.

\subsection{Results Discussion}

Case 1. The maximum positive uncertainties $\Delta_{C_{L}}=0.2 C_{L}$, $\Delta_{C_{D}}=0.2 C_{D}, \Delta_{C_{T}}=0.2 C_{T}$, and $\Delta_{C_{M}}=0.2\left[C_{M}(\alpha)+\right.$ $\left.C_{M}\left(\delta_{e}\right)+C_{M}(q)\right]$ are used here. In the case, the simulation results are provided in Figures 1 and 2. Specifically, the response curves for velocity and altitude are shown in Figures 1(a) and 1(b), in which it can be seen that the tracking is achieved in finite time with little overshoot. The results in Figures 1(c)-1(e) show the variations of AOA, FPA, and pitch rate, whereas the behavior of adaptive gain is provided in Figure 1(f). It can be observed from Figure 1(f) that adaptive gain $L(t)$ increases when $\sqrt{s_{v}^{2}+s_{h}^{2}}$ exceeds tolerance constant $\varepsilon=0.001$. In addition, the sliding manifolds for velocity $s_{v}$ and altitude $s_{h}$ in (20) are plotted in Figures 2(a) and 2(b). From that, it can be seen that the proposed control method is able to guide $s_{v}$ and $s_{h}$ to zero in the presence of bounded but unknown uncertainty. Figures 2(e) and 2(f) show the curves of derivatives in velocity and altitude errors, respectively. The results illustrate that not only the errors for velocity and altitude but also their derivatives converge to zero in finite time. The control variables, fuel equivalence ratio, and elevator deflection are given in Figures 2(c) and 2(f) where the results demonstrate that the control chattering is reduced effectively. In addition, the disturbance estimation values for $\Delta_{F_{y}}$ and $\Delta_{F_{h}}$ are added in Figure 3. Since the actual values for $\Delta_{F_{v}}$ and $\Delta_{F_{h}}$ are difficult to obtain, they are not provided in the simulation. However, the good tracking performance for velocity and altitude in Cases 1 and 2 to be given demonstrates the effectiveness of the control scheme in disturbance rejection.

Case 2. In order to further verify the effectiveness of the proposed control scheme in disturbance suppression, the 


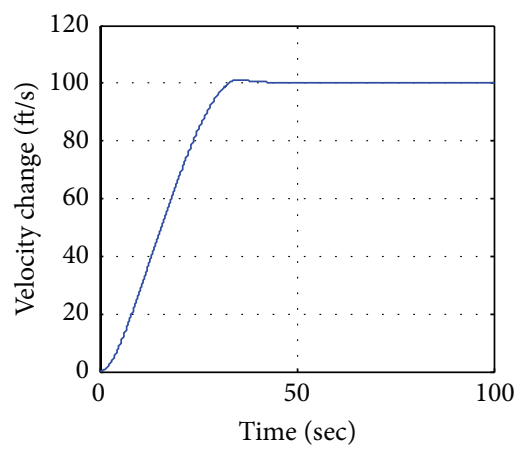

(a)

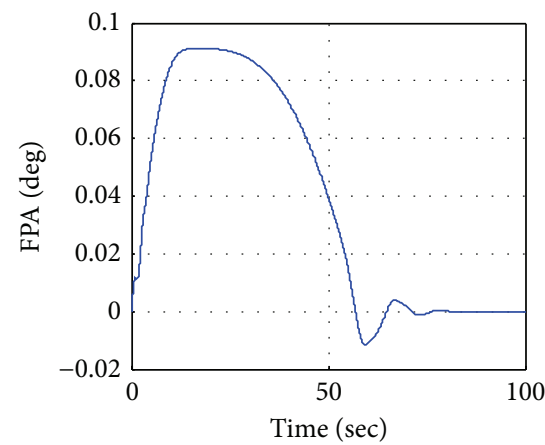

(d)

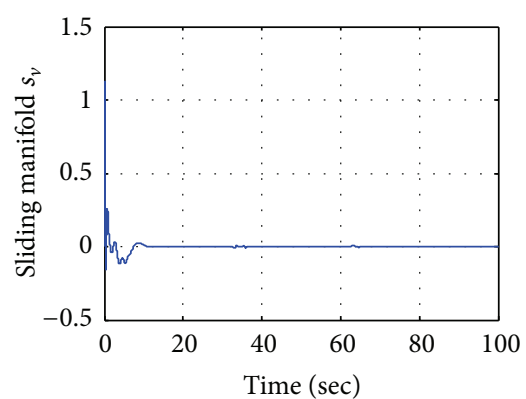

(a)

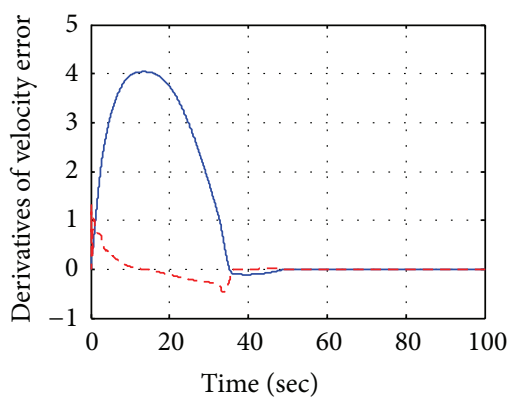

$-\mathrm{de}_{v}$ -.. dde

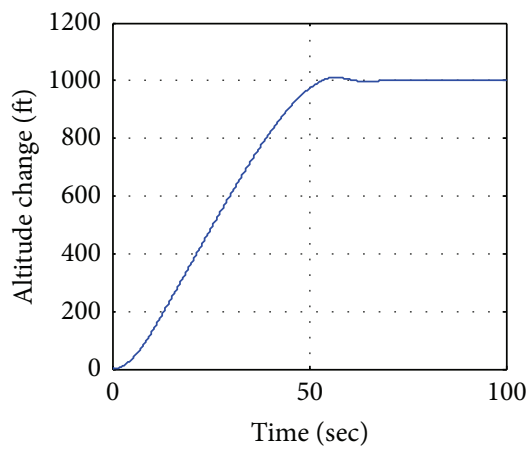

(b)

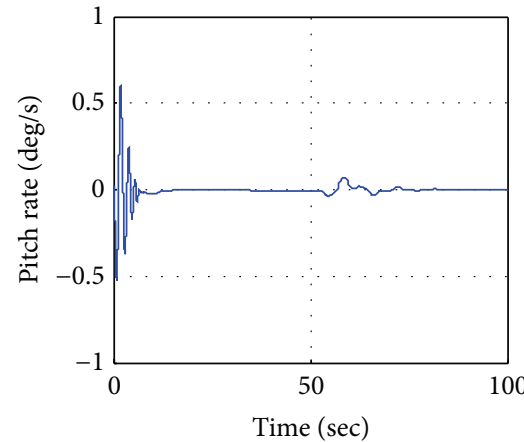

(e)

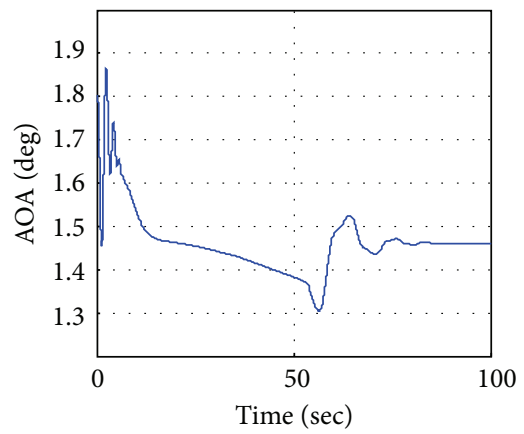

(c)

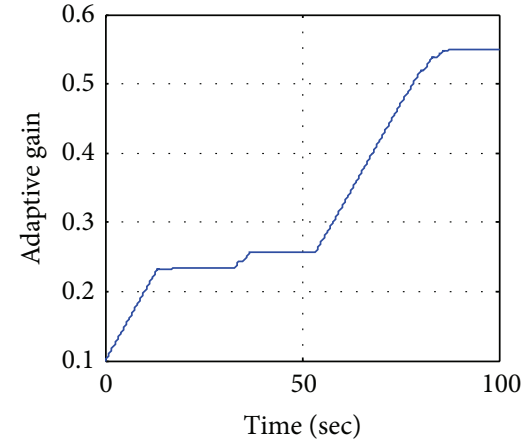

(f)

Figure 1: Flight states and adaptive law.

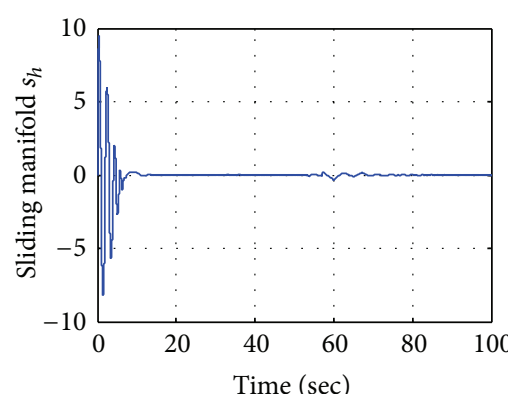

(b)

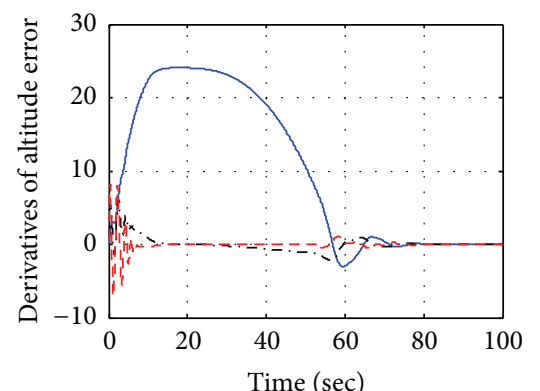

$-\mathrm{de}_{h}$

-..- $\mathrm{dde}_{h}$ -..- ddde $_{h}$

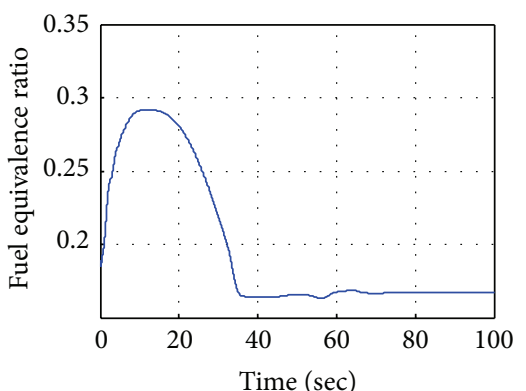

(c)

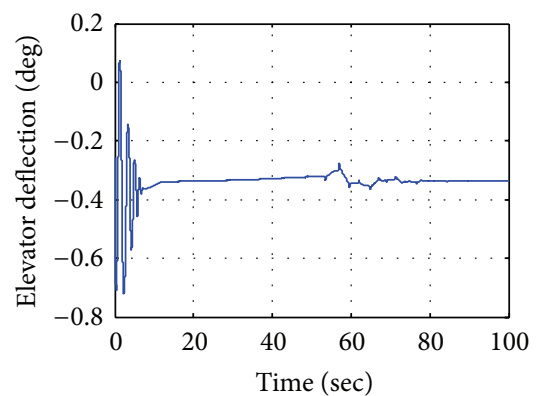

(f)

FIGURE 2: Flight controls and error information for velocity and altitude. 


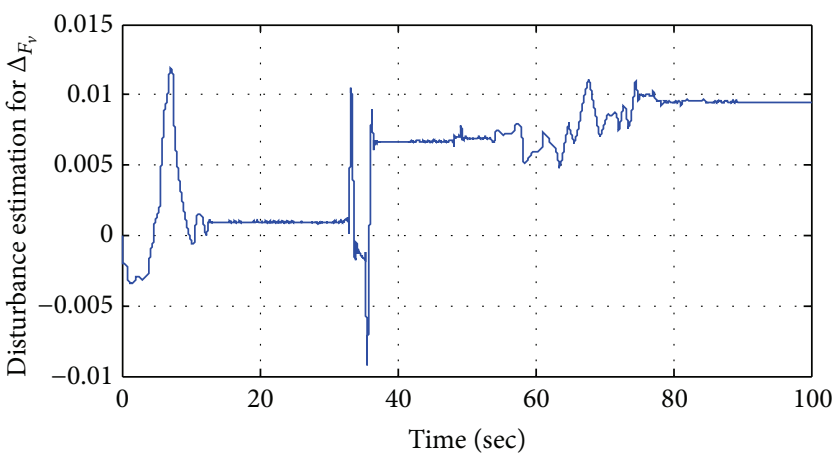

(a)

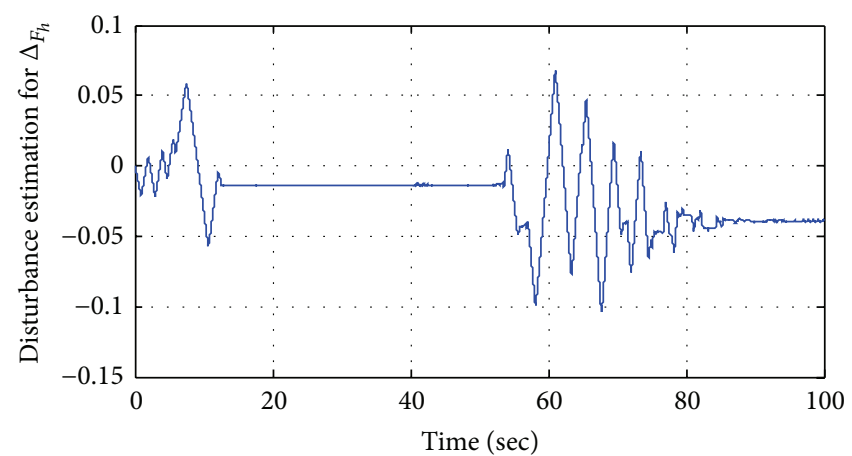

(b)

FIGURE 3: Disturbance estimated valued for $\Delta_{F_{v}}$ and $\Delta_{F_{h}}$.

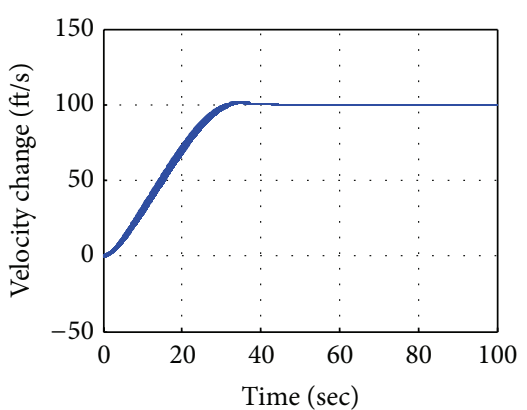

(a)

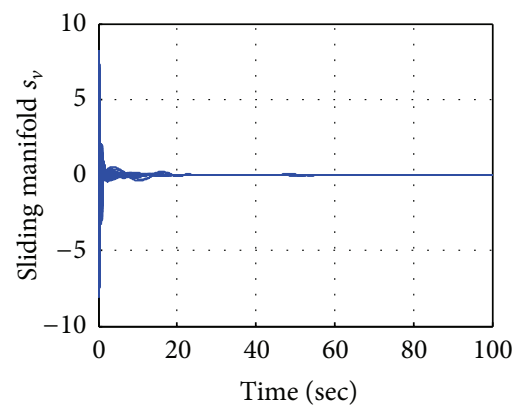

(d)

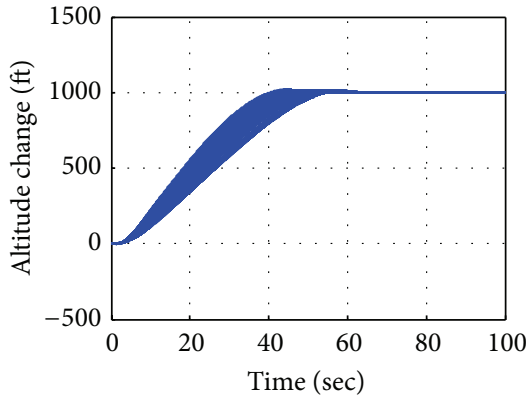

(b)

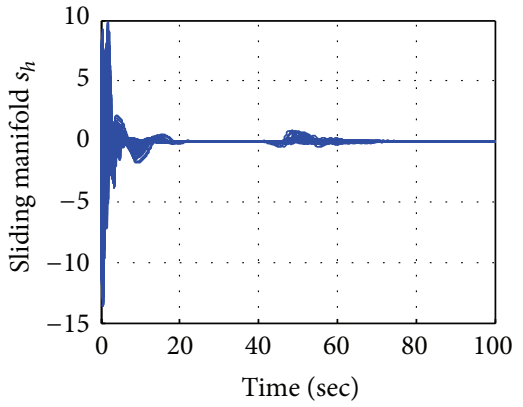

(e)

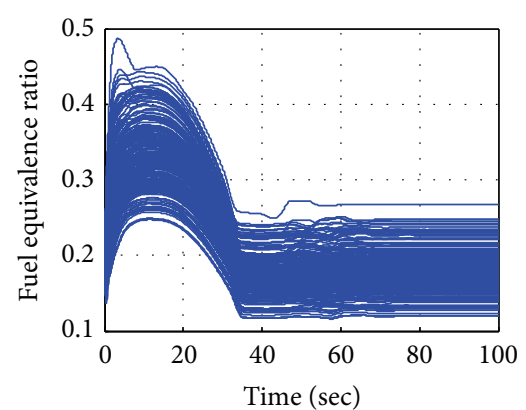

(c)

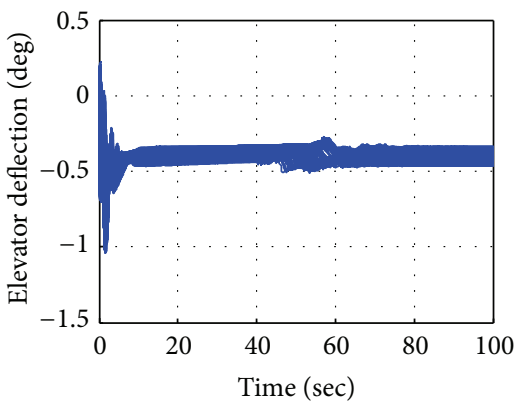

(f)

FIgURE 4: Monte Carlo simulation with 200 tests.

Monte Carlo simulation with 200 tests is conducted. The random uncertainty is added in the simulation according to the conditions in (5). All other parameters are the same as that provided in Case 1. In this case, the simulation results are provided in Figure 4. From Figures 4(a) and 4(b), it can be found that the velocity and altitude tracking can be achieved excellently even in the case of random uncertainty. The sliding manifolds for velocity as well as altitude and the corresponding control variables are also plotted in Figure 4. From Figures 4(c) and 4(d), it can be seen that the fuel equivalence ratio and elevator deflection have different steady-state values due to the effect of uncertainties. In fact, the magnitudes of fuel equivalence ratio and elevator deflection have to increase when positive drag force coefficient and negative lift force coefficient uncertainties are included in order to keep the stability of the system. Finally, the simulation results shown in this section demonstrate the effectiveness of the proposed control scheme in achieving the tracking for velocity and altitude.

\section{Conclusion}

The finite time tracking for hypersonic vehicle with boundary but unknown perturbations is discussed based on adaptive multivariable super-twisting control scheme. The finite time stability of the integrated controller and disturbance observer is guaranteed under the multivariable control architecture via Lyapunov analysis. The features of the proposed multivariable control scheme are gain adaptation, chattering reduction, nominal performance recovery, and finite time convergence. Finally, the Monte Carlo simulation with 200 tests is provided 
to demonstrate the effectiveness of the proposed control scheme.

\section{Competing Interests}

The authors declare that they have no competing interests.

\section{Acknowledgments}

This work has been supported by National Natural Science Foundation of China (61301244), Tianjin Research Program of Application Foundation and Advanced Technology (12JCZDJC30300), Aeronautical Science Foundation of China Supported by Science and Technology on Aircraft Control Laboratory (20125848004), Independent Innovation Fund of Tianjin University (2013XQ-0022), and Public Fund Supported by Tianjin Key Laboratory of Process Measurement and Control (TKLPMC-201315).

\section{References}

[1] D. K. Schmidt, "Optimum mission performance and multivariable flight guidance for airbreathing launch vehicles," Journal of Guidance, Control, and Dynamics, vol. 20, no. 6, pp. 1157-1164, 1997.

[2] K. P. Groves, D. O. Sigthorsson, A. Serrani et al., "Reference command tracking for a linearized model of an air-breathing hypersonic vehicle," in Proceedings of the AIAA Guidance, Navigation, and Control Conference and Exhibit, AIAA-20056144, San Francisco, Calif, USA, August 2005.

[3] H. D. Hughes, LPV Control for the Longitudinal Dynamics of a Flexible Air-Breathing Hypersonic Vehicle, Mechnaical Engineerin, North Carolina State University, Raleigh, NC, USA, 2010.

[4] D. O. Sigthorsson, P. Jankovsky, A. Serrani, S. Yurkovich, M. A. Bolender, and D. B. Doman, "Robust linear output feedback control of an airbreathing hypersonic vehicle," Journal of Guidance, Control, and Dynamics, vol. 31, no. 4, pp. 1052-1066, 2008.

[5] D. J. Leith and W. E. Leithead, "Survey of gain-scheduling analysis and design," International Journal of Control, vol. 73, no. 11, pp. 1001-1025, 2000.

[6] Q. Zong, Y. H. Ji, F. L. Zeng, and H. Liu, "Output feedback backstepping control for a generic hypersonic vehicle via small-gain theorem," Aerospace Science and Technology, vol. 23, no. 1, pp. 409-417, 2012.

[7] T. A. Adami, J. J. Zhu, M. A. Bolender, D. B. Doman, and M. Oppenheimer, "Flight control of hypersonic scramjet vehicles using a differential algebraic approach," in Proceedings of the AIAA Guidance, Navigation, and Control Conference, pp. 36803698, Keystone, Colo, USA, August 2006.

[8] Q. Wang and R. F. Stengel, "Robust nonlinear control of a hypersonic aircraft," Journal of Guidance, Control, and Dynamics, vol. 23, no. 4, pp. 577-585, 2000.

[9] L. Fiorentini, A. Serrani, M. A. Bolender, and D. B. Doman, "Robust nonlinear sequential loop closure control design for an air-breathing hypersonic vehicle model," in Proceedings of the American Control Conference, pp. 3458-3463, Seattle, Wash, USA, June 2008.

[10] L. Fiorentini, A. Serrani, M. A. Bolender, and D. B. Doman, "Nonlinear robust adaptive control of flexible air-breathing hypersonic vehicles," Journal of Guidance, Control, and Dynamics, vol. 32, no. 2, pp. 401-416, 2009.

[11] X. H. Yu and O. Kaynak, "Sliding-mode control with soft computing: a survey," IEEE Transactions on Industrial Electronics, vol. 56, no. 9, pp. 3275-3285, 2009.

[12] S. D. Gennaro, J. R. Dominguez, and M. A. Meza, "Sensorless high order sliding mode control of induction motors with core loss," IEEE Transactions on Industrial Electronics, vol. 61, no. 6, pp. 2678-2689, 2014.

[13] Y. Feng, X. H. Yu, and F. L. Han, "High-order terminal slidingmode observer for parameter estimation of a permanentmagnet synchronous motor," IEEE Transactions on Industrial Electronics, vol. 60, no. 10, pp. 4272-4280, 2013.

[14] H. Xu, M. D. Mirmirani, and P. A. Ioannou, "Adaptive sliding mode control design for a hypersonic flight vehicle," Journal of Guidance, Control, and Dynamics, vol. 27, no. 5, pp. 829-838, 2004.

[15] S. H. Li, S. H. Ding, and Q. Li, "Global set stabilisation of the spacecraft attitude using finite-time control technique," International Journal of Control, vol. 82, no. 5, pp. 822-836, 2009.

[16] A. Levant, "Homogeneity approach to high-order sliding mode design," Automatica, vol. 41, no. 5, pp. 823-830, 2005.

[17] Q. Zong, J. Wang, and Y. Tao, "Adaptive high-order dynamic sliding mode control for a flexible air-breathing hypersonic vehicle," International Journal of Robust and Nonlinear Control, vol. 23, no. 15, pp. 1718-1736, 2013.

[18] B. L. Tian, W. R. Fan, Q. Zong, J. Wang, and F. Wang, "Adaptive high order sliding mode controller design for hypersonic vehicle with flexible body dynamics," Mathematical Problems in Engineering, vol. 2013, Article ID 357685, 11 pages, 2013.

[19] J. Yang, S. Li, C. Sun, and L. Guo, "Nonlinear-disturbanceobserver-based robust flight control for airbreathing hypersonic vehicles," IEEE Transactions on Aerospace and Electronic Systems, vol. 49, no. 2, pp. 1263-1275, 2013.

[20] H. Sun, S. Li, and C. Sun, "Finite time integral sliding mode control of hypersonic vehicles," Nonlinear Dynamics, vol. 73, no. 1-2, pp. 229-244, 2013.

[21] J. Yang, S. Li, and X. Yu, "Sliding-mode control for systems with mismatched uncertainties via a disturbance observer," IEEE Transactions on Industrial Electronics, vol. 60, no. 1, pp. 160-169, 2013.

[22] I. Nagesh and C. Edwards, "A multivariable super-twisting sliding mode approach," Automatica, vol. 50, no. 3, pp. 984-988, 2014.

[23] S. Y. Khoo, L. H. Xie, S. K. Zhao, and Z. Man, "Multi-surface sliding control for fast finite-time leader-follower consensus with high order SISO uncertain nonlinear agents," International Journal of Robust and Nonlinear Control, vol. 24, no. 16, pp. 2388-2404, 2014.

[24] S. P. Bhat and D. S. Bernstein, "Geometric homogeneity with applications to finite-time stability," Mathematics of Control, Signals, and Systems, vol. 17, no. 2, pp. 101-127, 2005.

[25] Y. B. Shtessel, M. Taleb, and F. Plestan, "A novel adatpve-gain supertwisting sliding mode controller: methodology and application," Automatica, vol. 48, no. 5, pp. 759-769, 2012.

[26] J. X. Liu, S. Laghrouche, M. Harmouche, and M. Wack, "Adaptive-gain second-order sliding mode observer design for switching power converters," Control Engineering Practice, vol. 30, pp. 124-131, 2014. 
[27] B. Tian, L. Yin, and H. Wang, "Finite-time reentry attitude control based on adaptive multivariable disturbance compensation," IEEE Transactions on Industrial Electronics, vol. 62, no. 9, pp. 5889-5898, 2015.

[28] H. K. Khalil, Nonlinear Systems, Prentice Hall Press, New York, NY, USA, 3rd edition, 2001.

[29] A. Levant, "Higher-order sliding modes, differentiation and output-feedback control," International Journal of Control, vol. 76, no. 9-10, pp. 924-941, 2003. 


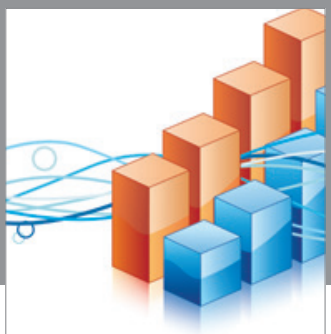

Advances in

Operations Research

vatem alat4

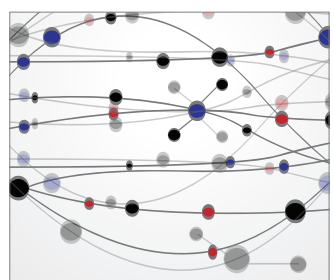

\section{The Scientific} World Journal
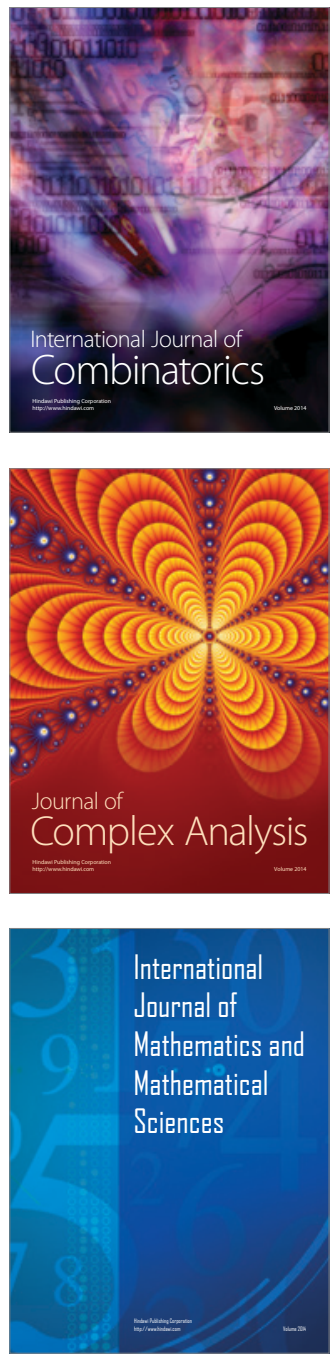
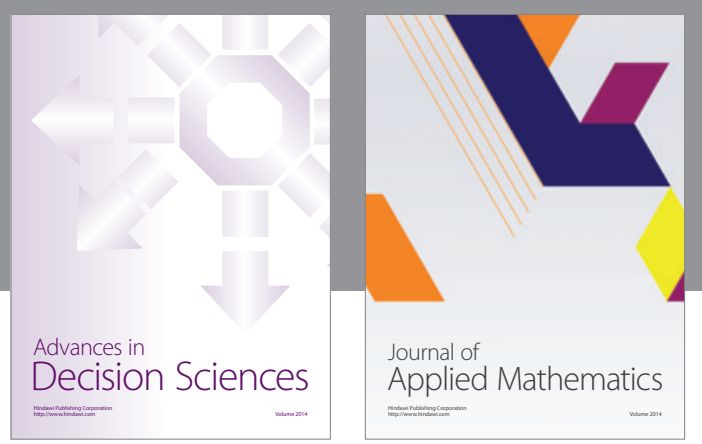

Algebra

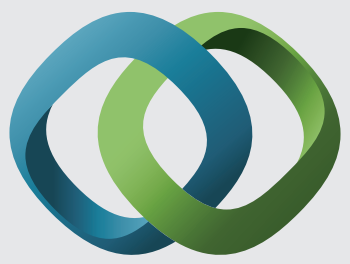

\section{Hindawi}

Submit your manuscripts at

http://www.hindawi.com
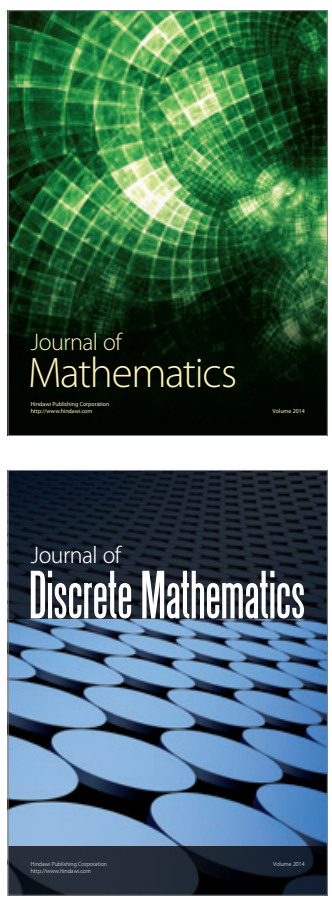

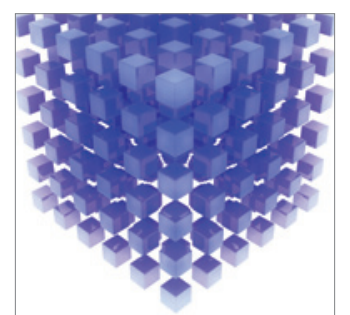

Mathematical Problems in Engineering
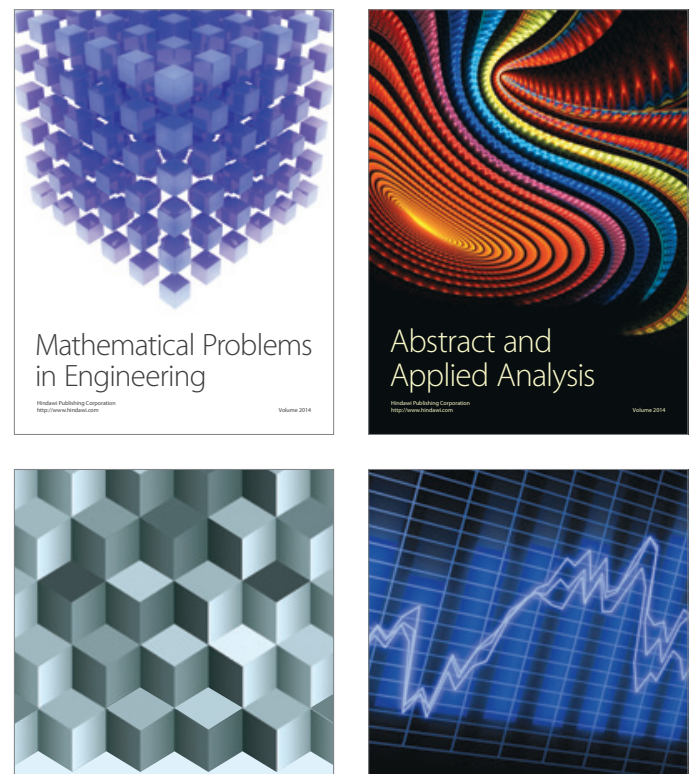

Journal of

Function Spaces

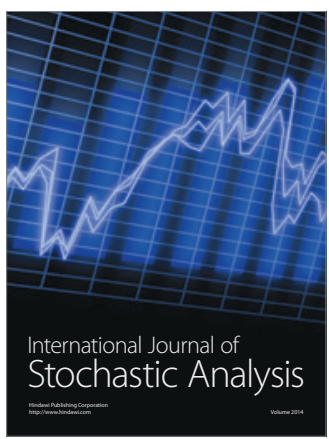

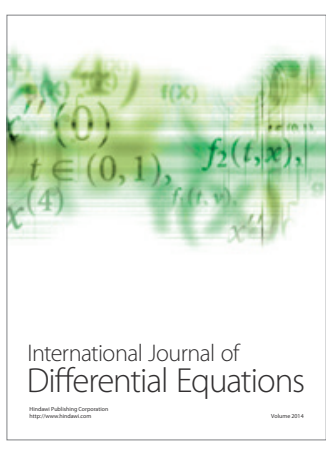
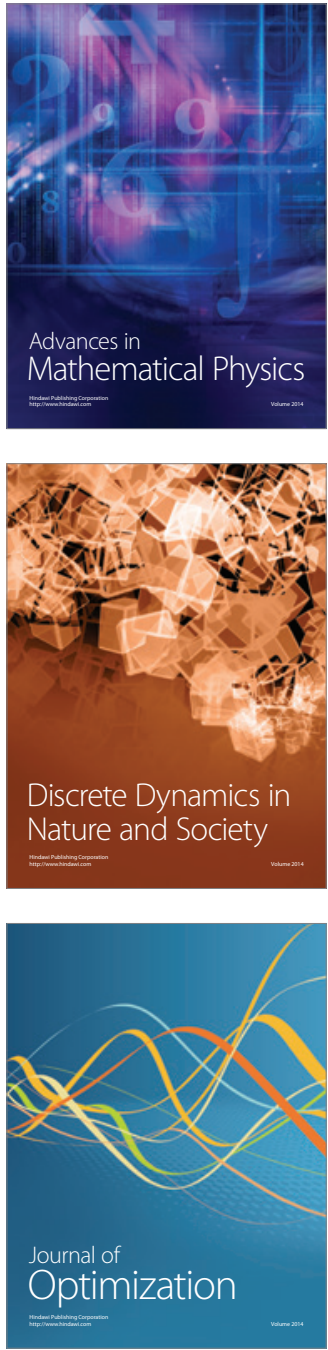